\title{
Quantum Size Effects in Nanoscale Metallic Structures
}

\author{
Dongmin Chen \\ The Rowland Institute at Harvard, Harvard University, Cambridge, MA02142 \\ And \\ Toshishige Yamada \\ NASA Ames Research Centre, Moffett Field, CA94035
}

\begin{abstract}
Spatial confinement of electrons in a nanoscale metallic structure gives rise to a set of standing wave states and quantized energy spectra. The quantized nature of the electrons and the enhanced boundary scattering could seriously affect the electron transport in future nanoscale interconnects. As revealed by scanning tunnelling microscopy, such quantum size effect also plays a competing role along side the classical thermodynamic effect in the shape relaxation of a metallic nanostructure. In particular, it leads to very unusual mass transport behaviours. Like the electron migration, such effect may have significant impact on the stability of nanoscale metal interconnects.
\end{abstract}

\section{INTRODUCTION}

As device features continue to shrink down to nanometer scale, it is widely anticipated that novel quantum mechanical properties of device structures and materials will affect device behaviour, operational principles and concept. Under spatial confinement, electron energies are quantized, leading to many novel effects, collectively known as quantum size effect (QSE). Electron transport in future nanoscale interconnects must be governed by these new quantum effects as well as the enhance boundary scattering. It has become increasingly evident that QSE also plays a significant role in shaping a small-scale structure where de Broglie wavelength is comparable its size[1-4].

Structural stability of a nanostructure is an important issue as the sizes of solid state devices continue to decrease. In general, a structure fabrication process, such as solid vapour deposition, can be far from equilibrium and postdeposition transformation of a metastable structure into a thermodynamically equilibrium shape will take place if kinetic barriers can be overcome as a result of heating or electron migration. This tendency is heightened for nanoscale structures as the surface/volume ratio increases and the dominant surface energy tries to minimize itself via shape change. For a large strained structure, its stability can be well described by the balance between surface tension and the bulk elastic energy based on the classical continuum theory [5-9]. In a nanoscale structure, quantized energy spectrum depends not only on the sizes but also on the shape, or the boundary conditions. It turns out that variation of the total electronic energy due to shape transformation is comparable to the associate change of the surface energy and bulk elastic energy. This can lead to very unusual mass transport behaviours. Like the electron migration, such effects may have serious impact on the stability of nanoscale metal interconnects.

\section{QUANTIZED STATES IN NANOSCALE METALLIC STRUCUTRES}

In nanoscale metallic structures, such as future interconnects, standing wave states can form readily inside
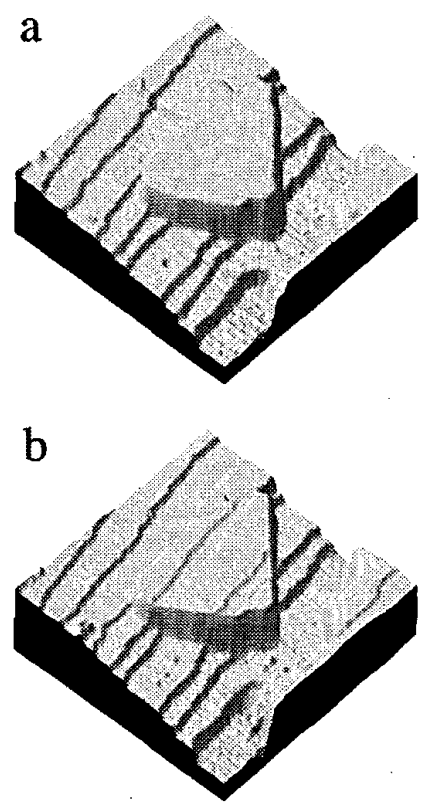

C

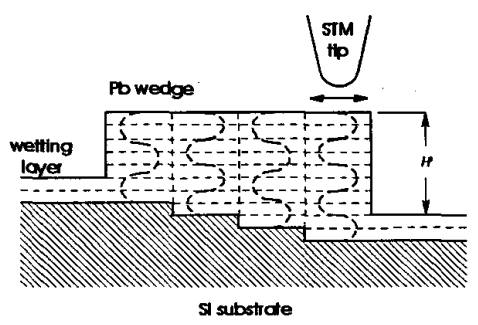

Figure 1. (a) $5000 \AA \times 5000 \AA \mathrm{STM}$ topography of a Pb wedge island grown on a stepped $\mathrm{Si}(111)$ surface descending from left to right. The thickness of the wedge increases successively from 8 to 12 layers. (b) STM image of the same wedge taken with an opposite polarity of the tip bias $(+5 \mathrm{~V})$, revealing the interference fringes of the highest occupied quantum state near the Fermi level. Both images were acquired at $4.8 \mathrm{~K}$. (c) a schematics illustrates the $\mathrm{Pb}$ wedge island. 
the metals, giving rise to the electron interference fringes on the surface of the nano-metallic structure. These quantum effects can be revealed with an atomic accuracy using low temperature scanning tunnelling microscopy (STM) and spectroscopy(STS)[10,11]. Fig. la is a STM image of a $\mathrm{Pb}$ wedge island grown on a $\mathrm{Si}$ substrate taken with a large negative tip bias with respect to the sample. $\mathrm{Pb}$ is insoluble in $\mathrm{Si}$ and on a Si surface $\mathrm{Pb}$ forms [111] oriented crystallites following the initial growth of two wetting layers. The $\mathrm{Pb}$ wedge expands over five substrate terraces. Despite the stepped substrate, the top surface of the $\mathrm{Pb}$ island is atomically flat and as the Si substrate descends down from left to right, the number of $\mathrm{Pb}$ layers on each terrace increases successively from 8 to 12, as illustrated in Fig. 1c.

When the tip bias polarity is flipped to positive (Fig. 1b), the STM image of the wetting layer region remains the same but the top of the wedge exhibits a set of up-and-down fringes which are precisely aligned with the substrate steps. A simple interpretation of these fringes can be realized if we examine the relationship between the Fermi wavelength and lattice spacing of $\mathrm{Pb} . \lambda_{F}=3.7 d_{0}\left(d_{0}=2.86 A\right)$, or $d_{0} \approx \lambda_{F} / 4$. The variation in the thickness by a single $\mathrm{Pb}$ layer will, therefore, change the interference condition for the Fermi electrons from destructive to constructive or vice versa. The discrete nature of the $\mathrm{Pb}$ wedge should give rise to a binary interference pattern observed in the experiment.

The underlying physics for the spontaneous formation of the interference fringes on a quantum wedge lies in the formation of the standing wave and discrete energy states as a result of the spatial confinement. Fig. 2a displays a sequence of $\mathrm{I}-\mathrm{V}$ spectra acquired with the STM tip placed successively above each fringe of a larger $\mathrm{Pb}$ wedge. The index next to each $\mathrm{I}-\mathrm{V}$ curve gives the number of the $\mathrm{Pb}$ layers, measured with respect to the top of the surrounding wetting layer. The staircase like spectra reflects the energy quantization of the standing wave states. The energy separation between two adjacent steps scales inversely with the number of atomic layers in that portion of the island, as shown in Fig. 2b[10,11].

Formation of quantum well states normal to a thin metal film is a consequence of the transverse confinement. In general, electrons in the plane remain to be extended Bloch waves and their energy is dispersed. Under a special circumstance where the effective mass of electron along the plane is much larger than that normal to the plane, as in the case of $\mathrm{Pb}$, quantized states do not overlap and QSE is greatly enhanced[12].

Several metals such $\mathrm{Ag}$, $\mathrm{Au}$ and In also exhibit quantum well sates. Work is underway to understand how do the energy quantization and boundary scattering affect the transport behaviour which is essential in VLSI interconnects.

\section{QUANTUM SIZE EFFECT ON STABILITY OF A NANOSCTRUCTURE}

Energy quantization relates intimately to the boundary conditions, both size and shape. This implies that QSE can

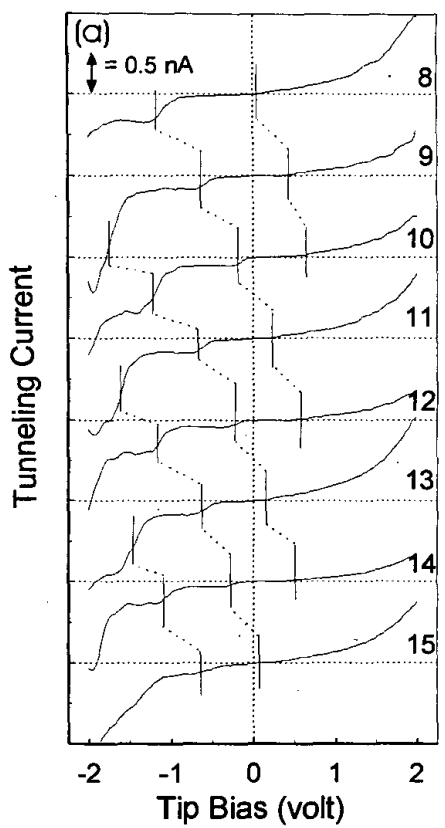

(b)

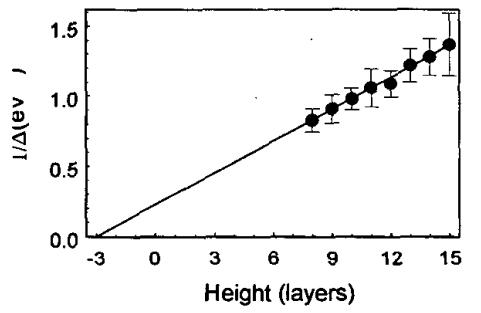

Figure 2. (a) A series of $I-V$ spectra (a) measured at $4.8 \mathrm{~K}$ over the fringe on the surface of the $\mathrm{Pb}$ wedge. The integers indicate the number of $\mathrm{Pb}$ layers above the surrounding wetting layer. Short vertical bars trace the progression of the states with the same quantum number $n$. (b) The inverse of the separation (filled circles) between two steps near zero bias in the $I-V$ curves of Fig. 4 as a function of the thickness of the wedge, measured in number of layers with respect to the surrounding $\mathrm{Pb}$ wetting layer. The solid line is a fit of

$1 / \Delta=H / \pi \hbar v$, to the data with $v,=1.9 \times 10^{\circ} \mathrm{cm} / \mathrm{sec}$.

affect the stability of a nanoscale structure. This is in strong contrast to larger structures where thermodynamics governs the shape. Indeed, the QSE plays a competing role along side the classical thermodynamic effect in the shape relaxation of a small metallic structure[13]. Together, these effects transforms a lead $(\mathrm{Pb})$ island from its initially flattop faceted morphology to a peculiar ring-shape island as shown in Figure 3. This process can be initiated or catalysed by the tip electric field of a scanning tunnelling microscope (STM). Even a single scan of the STM over the flat top island can trigger its shape transformation. Once the process starts, it is found that there is no need for further STM scanning. The resultant ring island has more steps, kinks, surface area, and is opposite to what thermodynamics would favour, i.e. a compact island. 

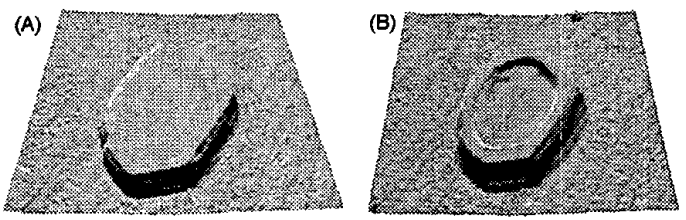

Figure 3. STM images $(8000 \AA x 8000 \AA)$ showing an initially flattop $\mathrm{Pb}$ island (a) grown on a $\mathrm{Si}(111)$ substrate transforms into a ring shape island (b). The height of the flattop island (a) is $-60 \AA$. The height of the ring is about $25 \AA$ measured with respect to the plateau of the central opening which is essentially at the same height as the flat island (a)

In addition, there exists a novel mass transport behaviour. Figure 4 is a series of STM images of the interior bottom surface of a ring island acquired consecutively at $3 \mathrm{~min}$. apart. In these images the $\mathrm{Si}$ substrate covered by $\mathrm{Pb}$ wetting layers descends from lower left towards upper right corner: Thus the number of $\mathrm{Pb}$ layers between the interior plateau and the substrate increases successively from lower left to upper right. In frame. (a), two patches of a new layer above the same substrate step is growing towards each other from the opposite side of the internal wall of the ring. We refer to this as the $N t h$-strip to indicate that it is on top of $N \mathrm{~Pb}$ layer. As we proceed to frame (b) the $N$ th strip nearly completes. Quite surprisingly, now the $(N+2)$ th strip also started to grow in the same manner, bypassing completely the $(N+1)$ th strip! As the $(N+2)$ th strip near completion, the $(N+4)$ th strip begins to grow, again bypassing the $(N+3)$ th strip (see Frame (c)). Notice that now the $(N+1)$ th strip also starts to grow, but with two layers at once rather than a single layer. The growth rate of the double layer strip is much slower than that of the single layer strip. Consequently, frame (d) changes very little from (c).

To summarize, there exist novel strip-flow growth and double-step growth on selective strips of the plateau inside the ring, defined by the substrate steps more than $60 \AA$ below. These peculiar mass transport behaviours cannot be explained by know classical mechanisms. The fact that the strips are precisely correlated to the substrate steps, as do the interference fringes, indicates that the boundary condition somehow controls where the mass must flow and accumulate on the surface.
From preceeding section, we see that a set of quantized states(QS) (or 2D subbands) as a function of the metal layers must present inside the island. The number of the total QS increases by one with the addition of each new layer in the island. This causes a reduction of the level separation $\Delta$ and a shift of the QS near $E_{F}$ by $\delta=2 l \Delta / \lambda_{F}$, where $\lambda_{F} \approx 3.7 l(l=2.86 \AA$, is the $\mathrm{Pb}(111)$ interlayer spacing): Around 20 layers (typical height of the inner plateau of the ring), the highest occupied QS is farther away from $E_{F}$ in strips with even $N$ than that in strips with odd $N$. This suggests that the former would give rise to lower system energy. The inset of Fig. 5 shows a set of Fermi disks enclosed in the Fermi sphere, representing a quantized free electron system bound by infinite potential burier. The correction due to the quantization of the electronic contribution to the surface energy is the sum of all the disks minus the equivalent total energy in a continuum case. As $N$ change from odd to even, the Fermi disks shift down by roughly a half of the disk-to-disk separation resulting in a change in surface energy. Moreover, it has been shown previously that the electron motion is highly anisotropic in the $\mathrm{Pb}$ overlayer and $m_{\|}^{*} / m_{\perp}^{*} \geq 30$ [12]. In another words the Fermi surface is quite flat near $\Gamma$-L and the equi-energy surface in the $k$-space become highly distorted. Under this limit that the width of the 2D subband is smaller than the band-to-band separation, the total electron energy, a sum of a set of occupied flat bands, exhibits a parity effect, i.e. the oscillatory dependence on odd vs. even $N$ as plotted in Figure 5.

Thus the strip-flow growth on the odd number layer is to convert the strip with higher surface energy to an even number layer with a lower surface energy. The energy gain becomes smaller as it moves sequentially from the thinner side to the thick side. When all the strips are in the low energy state (even number of layers in this case), subsequent growth on such region will take place by a double layer strip flow, so to maintain the low surface energy. This process is much less probable as it requires two atoms arrive in the same place at the same time. It appears that atoms diffusing on the plateau can clearly "sense" the quantized energy states inside the island and preferentially attach to regions that further reduces the surface energy, limiting its own growth. In general, once the plateau inside the ring reaches this low energy phase, the surface now becomes difficult to wet and it respells the
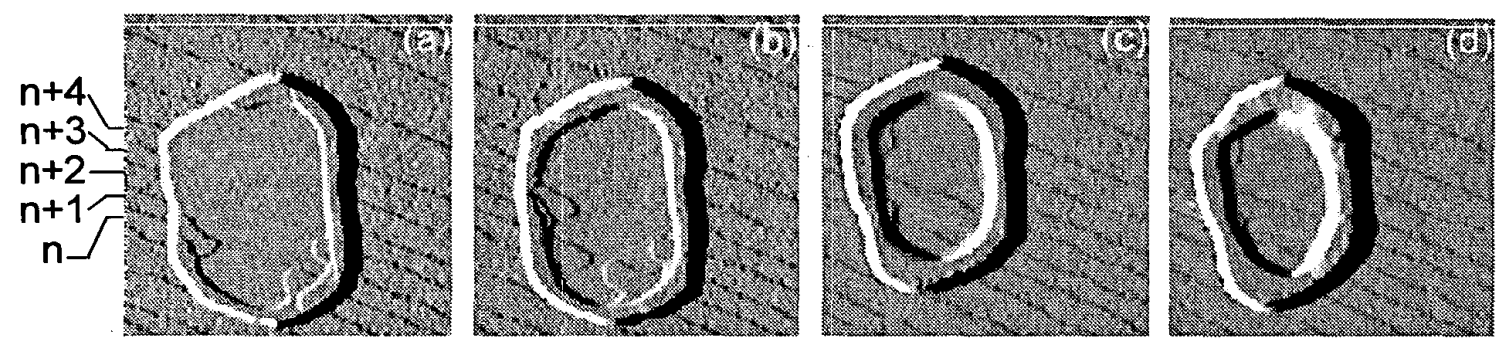

Figure 4. Sequentially recorded STM images $(8000 \AA \times 8000 \AA)$ showing an unusual sequential strip-flow growth on alternating strips on the plateau inside the ring. 


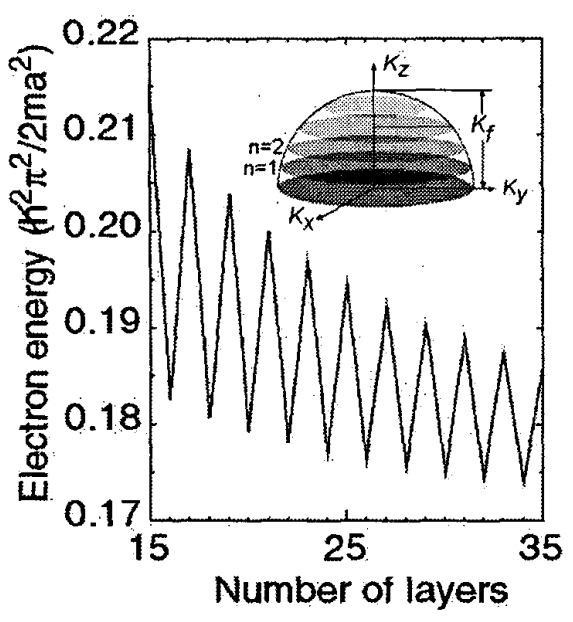

Figure 5. Electron energy per unit area as a function of number of layers for a quantized system with large anisotropic effective masses $\left(m_{\perp}^{*} \square m_{\|}^{*}\right), a$ being the in plane lattice constant. Inset shows a set of Fermi disks of a free electron system.

arriving atoms and drives them to the inner wall of the ring hence stabilize the ring shape which would other wise disintegrate rapidly.

\section{SUMMARY AND DISCUSSIONS}

Metal interconnects are an essential part of VLSI circuitry. A microprocessor toady contains as many as eight layers of interconnects. When devices are scaled down, the interconnect lines must also be scaled down in all dimensions, width, length, thickness, spacing, and insulator thickness [14]. The QSE is irrelevant in interconnects of microelectronics since the Fermi wave length in a metal $(\sim$ $1 \mathrm{~nm})$ is much shorter than that in a semiconductor $(\sim 50$ $\mathrm{nm}$ ). For this reason, the QSE has been discussed only for semiconducting devices, such as Metal-OxideSemiconductor Field-Effect Transistors (MOSFET). Inversion layer quantization will raise the threshold voltage of a MOSFET[14] since more band bending is required to populate the lowest subband, located somewhat above the bottom of the conduction band. This situation effectively increases the oxide thickness, reduces the oxide capacitance, and reduces the transconductance of the device.

However, the QSE in interconnect lines is no longer hypothetical in nanoelectronics, especially when the Fermi wave length is long $(\sim 1 \mathrm{~nm})$ with a strong mass anisotropy like our case. Then, the energy quantization in the thickness direction is observable as discussed in Sec. II, and will certainly affect the properties at the device contact. In fact, the position of the Fermi level is modified due to the QSE and the metallic work function is effectively modified. This will change the potential barrier at a metal-semiconductor contact. Thus, the conditions for a Schottky contact or an Ohmic contact must be re-examined. This work function modification will also be relevant for a gate electrode of a MOSFET when its thickness is reduced, and will again influence the threshold voltage according to a similar mechanism above. The current flow through the interconnect lines will mechanically change the line shape as discussed in Sec. III, and this is reminiscent of the electron migration problem [14]. These effects will be prevented if we do not scale in the thickness direction, but the standard scaling rule of interconnect lines in all directions is important for the appropriate behaviour of the circuitry and for the planar fabrication process. We need to stick to the scaling rule, and therefore, the QSE in the thickness direction in metallic lines is important in the future nanoelectronics.

In conclusion, the quantum confinement leads to discrete energy states of electrons in a nanoscale metal structure. The quantized energy. spectrum will have important consequences on electronics applications, including the effective modification of the work function. The quantum confinement also affects the mass transport and stability of the shape of the nanointerconnect lines. These observed effects will be quite universal for the interconnect lines in the future nanoelectronics.

\section{ACKNOWLEDGEMENT}

The authors wish to thank Dr. I Altfeder and Dr. H Okamoto for their invaluable contributions. This work was supported in part by the Rowland Institute at Harvard, and by a grant from NASA Ames Research Centre (Grant No. NCC 2-1312).

\section{REFERENCES}

[1] D. A. Luh, T. Miller, J. J. Paggel, et al., Science 292, 1131 (2001).

[2] Z. Zhang, Q. Niu, and C.-K. Shih, Phys. Rev. Lett. 80, 5381 (1998).

[3] V. Yeh, L. Berbil-Bautista, C. Z. Wang, et al., Phys. Rev. Lett. 85, $5185(2000)$.

[4] W. B. Su, S. H. Chang, W. B. Jian, et al., Phys. Rev. Lett, 86, 5116 (2001).

[5] R. J. Asaro and W. A. Tiller, Metall. Trans 3, 1789 (1972).

[6] M. A. Grinfeld, J. Nonlinear Sci. 3, 35 (1983).

[7] D. J. Sroloviz, Acta Metall 37, 621 (1989).

[8] B. J. Spencer, P. W. Voorhees, and S. H. Davis, Phys. Rev. Lett. 67, $3696(1991)$.

[9] J. Tersoff and R. M. Tromp, Phys. Rev. Lett. 70, 2782 (1993).

[10] I. B. Altfeder, K. A. Matveev, and D. M. Chen, Phys. Rev. Lett. 78, 2815 (1997)

[11] D. Chen, J. Electron Sepctroscopy 109, 85 (2000).

[12] I. B. Altfeder, D. M. Chen, and K. A. Matveev, Phys. Rev. Lett. 80 , 4895 (1998).

[13] H. Okamoto, H., D.M. Chen, T. Yamada, Phys. Rev. Lett. 89, 256101 (2002).

[14] Y. Taur and T.-H. Ning, Fundamentals of Modern VLSI Devices, (Cambridge, New York, 1998). 